\title{
Decreased serum cell-free DNA levels in rheumatoid arthritis
}

\author{
Marina Dunaeva ${ }^{1} \cdot$ Bastiaan C. Buddingh ${ }^{1} \cdot$ René E. M. Toes $^{2} \cdot$ Jolanda J. Luime $^{3}$. \\ Erik Lubberts $^{3}$ - Ger J. M. Pruijn ${ }^{1}$
}

Received: 15 April 2015/Accepted: 28 May 2015/Published online: 27 June 2015

(C) The Author(s) 2015

\begin{abstract}
Purpose Recent studies have demonstrated that serum/plasma DNA and RNA molecules in addition to proteins can serve as biomarkers. Elevated levels of these nucleic acids have been found not only in acute, but also in chronic conditions, including autoimmune diseases. The aim of this study was to assess cell-free DNA (cfDNA) levels in sera of rheumatoid arthritis (RA) patients compared to controls.

Methods cfDNA was extracted from sera of patients with early and established RA, relapsing-remitting multiple sclerosis patients (RRMS) and healthy subjects, and its concentration was determined by quantitative PCR using two amplicons, Alu115 and $\beta$-actin205, corresponding to Alu repetitive elements and the $\beta$-actin single-copy gene, respectively. Serum DNase activity was measured by a single radial enzyme diffusion method.

Results Reduced levels of cfDNA were observed in patients with established RA in comparison with healthy controls, early RA patients and RRMS patients. There were
\end{abstract}

Electronic supplementary material The online version of this article (doi:10.1007/s13317-015-0066-6) contains supplementary material, which is available to authorized users.

Ger J. M. Pruijn

G.Pruijn@ncmls.ru.nl

1284 Department of Biomolecular Chemistry, Institute for Molecules and Materials and Radboud Institute for Molecular Life Sciences, Radboud University Nijmegen, P.O. Box 9101, 6500 HB Nijmegen, The Netherlands

2 Department of Rheumatology, Leiden University Medical Center, Leiden, The Netherlands

3 Department of Rheumatology, Erasmus MC, University Medical Center Rotterdam, Rotterdam, The Netherlands no significant differences in cfDNA concentration between healthy controls, early RA and RRMS patients. Total DNase activity appeared to be similar in the sera of all tested groups.

Conclusions Our results demonstrate that cfDNA levels are strongly reduced in the sera of established RA patients, which is not caused by changes in DNase activity. Measurement of cfDNA can distinguish established RA patients from early RA patients. Thus, cfDNA may serve as a biomarker in RA.

Keywords Autoimmune diseases - Rheumatoid arthritis · Cell-free DNA · Biomarker

\section{Introduction}

Rheumatoid arthritis (RA) is a chronic heterogeneous autoimmune disease characterized by progressive joint destruction. Serological tests, most notably the detection of anti-citrullinated protein antibodies (ACPA) and rheumatoid factor (RF), play an important role in diagnosing RA. ACPA were found in up to $75 \%$ of RA patients [1]. RF is present in $60-90 \%$ of patients with established RA (esRA) and in up to $50 \%$ of patients with early RA (eRA) [2]. In contrast to ACPA the disease specificity of RF is relatively low, with 3-5\% of healthy adults showing the presence of $\mathrm{RF}$ in their serum, which increases to $10-30 \%$ in the elderly [3]. RF was also found in patients with other autoimmune and infectious diseases.

Earlier it was suggested that the sooner RA patients are treated the better their prognosis is [4]. Although the introduction of ACPA and RF tests has greatly contributed to improving the early diagnosis of RA, additional molecular markers are needed, e.g., to identify 
seronegative patients, to differentiate between disease phenotypes, to predict responsiveness to treatment and to improve prognosis.

Circulating cell-free DNA (cfDNA), which is defined as extracellular DNA occurring in blood serum or plasma, has been widely studied and is considered as a potential biomarker for the detection and monitoring of various human diseases such as stroke [7, 8], myocardial infarction [9], sepsis [10] and acute pancreatitis [11], as well as some chronic conditions such as cancer [12]. Significantly higher cfDNA levels were found in the serum of cancer patients with advanced disease stages or metastases [13] and it has been previously shown that circulating cfDNA carries tumor-specific genetic alterations [14].

Circulating cfDNA molecules are present in only limited amounts in blood of healthy individuals, since dying cells and remnants of dead cells are efficiently removed, mainly in the liver [5,6]. The origin and exact mechanism of cfDNA release are still controversial. Accumulation of cfDNA in plasma/serum might result from an excessive release of DNA caused by massive cell death, inefficient elimination of dead cells, extracellular DNA traps formed during inflammation, or a combination of these. Thus, the concentration of plasma/serum cfDNA might reflect the magnitude of cell damage and inflammation.

It is not known whether cfDNA can be used as a rapid and sensitive marker of RA. In analogy with cancer, elevated levels of circulating cfDNA have been hypothesized to occur in autoimmune diseases [15]. Alteration of cfDNA levels has been reported for autoimmune diseases such as systemic lupus erythematosus (SLE), systemic sclerosis, Sjögren's syndrome [16] and RA [17]. The aim of the study described here was to quantify cfDNA levels in sera of eRA and esRA patients using both single-copy gene and Alu repetitive element analyses.

\section{Method}

\section{Patients}

Sera from RA patients were obtained from four different centers. Samples from esRA patients were collected at the Radboud University Nijmegen Medical Center $(n=9)$, at the St. Maartenskliniek in Nijmegen $(n=10)$ and at the Catharina Hospital in Eindhoven $(n=8)$. Samples from 22 eRA patients were obtained from the Leiden Early Arthritis Clinic, an inception cohort of patients with recent onset arthritis (symptoms duration $<2$ years) that was started at the Department of Rheumatology of the Leiden University Medical Center in 1993 and was described in detail previously [18]. Samples from 20 eRA patients were collected at the Erasmus Medical Center in Rotterdam. As controls, a cohort of relapsing remitting multiple sclerosis patients (RRMS; collected at the Multiple Sclerosis Center Nijmegen; $n=44$ ), and a group of healthy control individuals (collected at the Sanquin Blood Bank in Nijmegen; $n=50$ ) were included. All RA patients fulfilled the American College of Rheumatology 1987 revised criteria for the classification of RA. Patient sera were used in accordance with the code of conduct of research with human material in The Netherlands. All subjects gave informed consent. Serum samples were aliquoted and stored at $-80{ }^{\circ} \mathrm{C}$ prior to further use. Patient characteristics are summarized in Supplementary Table S1.

\section{Serum cfDNA isolation and quantification}

Sera of 29 control subjects, 39 eRA, 26 esRA and 33 RRMS patients were used for cfDNA isolation. cfDNA was extracted from $200 \mu \mathrm{L}$ of serum using the QIAamp DNA Blood Mini kit (Qiagen) according to the manufacturer's manual and was eluted with $50 \mu \mathrm{L}$ of water. The amounts of isolated cfDNA were determined using real-time PCR analyses. Two different targets were chosen for quantification: a housekeeping gene, $\beta$-actin, and Alu repetitive elements. Three independent analyses were performed. Each PCR reaction was performed in a total volume of $10 \mu \mathrm{L}$ containing $1 \times$ GoTaq master mix (Promega), $0.75 \mu \mathrm{M}$ of each primer, and $0.5-1 \mu \mathrm{L}$ of cfDNA solution. The amplicons resulted in 115-bp (Alu) and 205-bp ( $\beta$ actin) fragments [see Table 1 for primer sequences; 19]. Cycle threshold $(\mathrm{Ct})$ values were measured with a StepOne Plus qPCR machine (software version 2.2; Applied Biosystems; Warrington, UK). Thermocycling was performed at $95{ }^{\circ} \mathrm{C}$ for $10 \mathrm{~min}$ ( $\beta$-actin205) or for $2 \mathrm{~min}$ (Alu115), followed by 40 cycles at $95{ }^{\circ} \mathrm{C}$ for $15 \mathrm{~s}$ and $60{ }^{\circ} \mathrm{C}$ for $60 \mathrm{~s}$. Each sample was analyzed in duplicate and negative controls were included in each run. Melting curve analysis was performed to confirm the specificity of PCR products. The absolute DNA concentration was calculated according to the standard curves obtained with parallel analyses of human genomic DNA (Qiagen) with concentrations varying from 0.005 to $10 \mathrm{ng} / \mu \mathrm{L}$.

The Ct values obtained for cfDNA samples were converted to serum cfDNA concentrations using the following formula [20]:

$[\mathrm{cfDNA}]=\frac{Q}{V_{\mathrm{PCR}}} \times \frac{V_{\mathrm{DNA}}}{V_{\mathrm{ext}}}$

with [cfDNA] being the cfDNA concentration, $Q / V_{\mathrm{PCR}}$ the cfDNA concentration as deduced from the standard curve, $V_{\mathrm{DNA}}$ the volume of the isolated cfDNA solution, and $V_{\text {ext }}$ the volume of serum used for cfDNA extraction. 
Table 1 Primers used for qPCR reaction

\begin{tabular}{lll}
\hline & Forward primers $\left(5^{\prime}-3^{\prime}\right)$ & Reverse primers $\left(5^{\prime}-3^{\prime}\right)$ \\
\hline Alu115 & CCTGAGGTCAGGAGTTCGAG & CCCGAGTAGCTGGGATTACA \\
$\beta$-actin205 & GAGACCTTCAACACCCCAGCC & GGATCTTCATGAGGTAGTCAG \\
\hline
\end{tabular}

\section{DNase activity assay}

Sera of 50 control subjects, 16 esRA and 44 RRMS patients were used for DNase activity assay. To determine serum total DNase activity, a single radial enzyme diffusion (SRED) method was used [21]. This method is based on the principle that DNases present in serum samples are able to diffuse radially through a thin layer of agarose, which contains DNA, react with the DNA and hydrolyze it. Agarose gels (1.4\%; MetaPhor Agarose, Lonza) were prepared in buffer containing $20 \mathrm{mM}$ Tris- $\mathrm{HCl}, \mathrm{pH} 7.5$, $2 \mathrm{mM} \mathrm{MgCl}_{2}, 2 \mathrm{mM} \mathrm{CaCl} 2,100 \mathrm{ng} / \mathrm{mL}$ DNA from herring testes (Sigma-Aldrich) and GelStar stain (Cambrex). Wells (approx. $0.5 \mathrm{~mm}$ diameter) were punched in the gel. A standard curve was generated by serial dilutions of DNase I enzyme (Qiagen). Two microlitre of DNase I solution (concentration series) or serum samples were added to the wells. The gels were incubated in a humidified dark chamber at $37^{\circ} \mathrm{C}$ for $24 \mathrm{~h}$. GelStar fluorescence was visualized with a Doc-Print II System (Vilbert Lourmat). DNase activity was quantified by measuring the diameter of the non-stained circles resulting from DNA hydrolysis.

\section{Statistical analysis}

A statistical comparison of the data for the different groups was performed using GraphPad Prism software. For nonparametric comparisons, univariate analysis by the MannWhitney $U$ test was performed. Differences were considered significant at $p<0.05$. The diagnostic sensitivity and specificity were determined by receiver operator characteristic (ROC) analysis.

\section{Results}

\section{Serum cfDNA levels}

To determine the levels of cfDNA in sera, a quantitative real-time PCR approach was chosen for two different amplicons, one for Alu repeats and another for the $\beta$-actin gene. In addition to sera from esRA patients, sera from healthy individuals and from RRMS patients were used as controls; RRMS was chosen as another autoimmune disease with a different disease onset and with different treatment strategies. Alu repeats are highly repetitive sequences, which are found in approximately $10^{6}$ copies in the human genome, whereas $\beta$-actin is encoded by a singlecopy gene. As shown in Figs. 1a and 2a, the cfDNA levels determined by the Alu amplicon (median $39 \mathrm{ng} / \mathrm{mL}$; range $10-403 \mathrm{ng} / \mathrm{mL}$ ) and by the $\beta$-actin amplicon (median $14 \mathrm{ng} / \mathrm{mL}$; range 6-37 $\mathrm{ng} / \mathrm{mL}$ ) appeared to be much lower in the sera from esRA patients $(n=26)$ than in those from healthy controls $(n=26)$ and from RRMS patients $(n=33)(p<0.0001)$. RRMS sera showed similar levels of cfDNA as the healthy control sera (Figs. 1a, 2a; median $233 \mathrm{ng} / \mathrm{mL}$ (range 47-6900 ng/mL) and $206 \mathrm{ng} / \mathrm{mL}$ (range 42-899 ng/mL), respectively, for Alu115, and $188 \mathrm{ng} / \mathrm{mL}$ (range 18-3804 ng/mL) and $177 \mathrm{ng} / \mathrm{mL}$ (range 31-920 ng/ $\mathrm{mL})$, respectively, for $\beta$-actin205).

To assess whether the Alu115- and $\beta$-actin205-based qPCR assay for cfDNA can serve to discriminate RA patients from control individuals, receiver operating characteristic (ROC) analysis was applied (Figs. 1b, 2b, respectively). This analysis revealed that the area under the curve (AUC) values were high (AUC $=0.914$ for Alu115 and $\mathrm{AUC}=0.994$ for $\beta$-actin205). It is important to note that the RA samples analyzed came from three distinct cohorts of esRA patients, which makes it unlikely that the differences observed are due to trivial factors associated with the handling and storage of the samples. The Alu115 and $\beta$-actin205 qPCR analyses do not seem to be useful to discriminate RRMS patients from healthy controls.

Previously, ACPA have been reported to discriminate between two distinct populations of RA patients [22, 23]. Therefore, we also compared cfDNA levels in patients with anti-cyclic citrullinated peptide (anti-CCP)-positive RA and anti-CCP-negative RA. No significant differences were observed between the levels of cfDNA in anti-CCP2-positive and anti-CCP2-negative RA sera (Fig. 3).

The early diagnosis of RA and start of treatment is beneficial for patients to prevent or abrogate ongoing joint damage. Biomarkers which facilitate the early diagnosis of RA are needed in addition to ACPA. To study whether cfDNA levels were already decreased at the early stages of the disease, we determined cfDNA levels also in sera from eRA patients $(n=34)$ using the Alu115 and $\beta$-actin205 primer sets and performed additional analyses with material from healthy controls $(n=29)$. The results demonstrated that there was no significant difference between serum cfDNA levels of healthy individuals and eRA patients (Fig. 4). Comparison of eRA $(n=39)$ and esRA ( $n=24)$ samples, however, showed much lower serum cfDNA levels for esRA patients than the levels in the eRA 


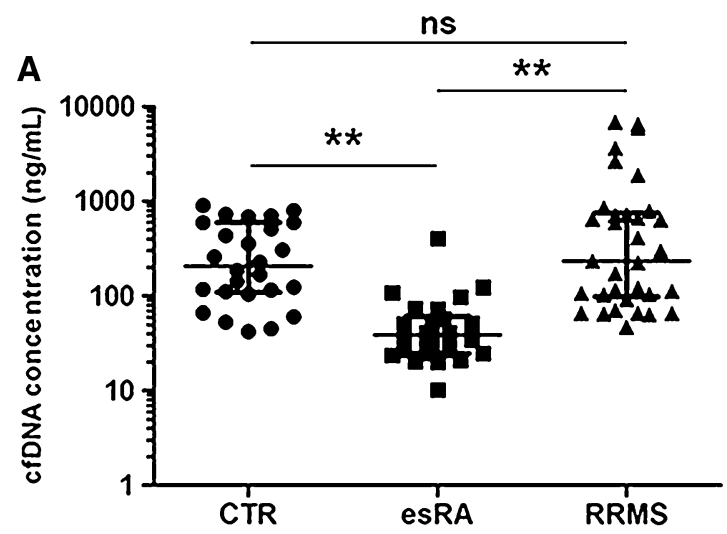

Fig. 1 Alu repeat-based detection of serum cfDNA. DNA was isolated from sera of healthy individuals (CTR), RA and RRMS patients and subjected to qPCR analysis with primers for the Alu115

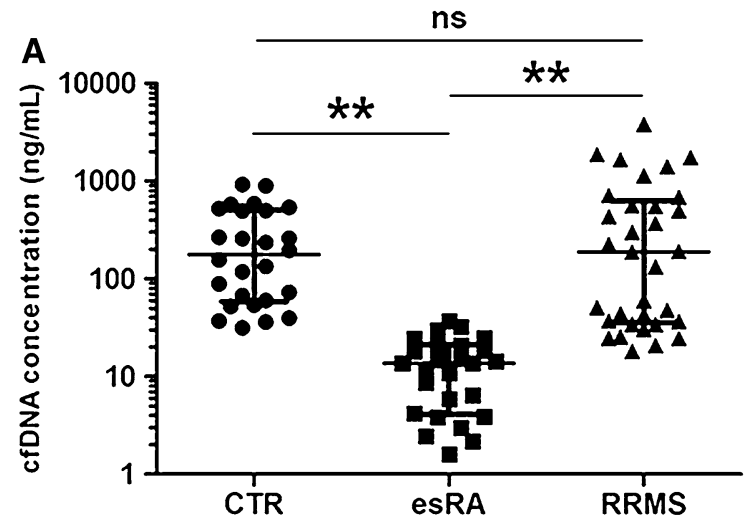

Fig. $2 \beta$-actin gene-based detection of serum cfDNA. DNA isolated from sera of healthy individuals (CTR), RA and RRMS patients was subjected to qPCR analysis with primers for the $\beta$-actin205 amplicon

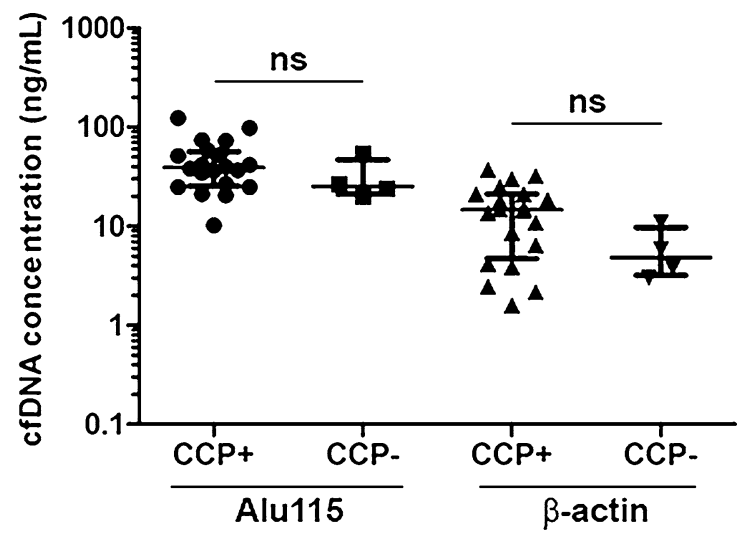

Fig. 3 cfDNA concentration does not correlate with anti-CCP2 autoantibody status. Comparison of circulating Alu115 and $\beta$ actin205 DNA in sera from patients with anti-CCP2-positive $(\mathrm{CCP}+)$ and anti-CCP2-negative $(\mathrm{CCP}-) \mathrm{RA}$. The bars indicate the median with interquartile range. ns indicates not significant

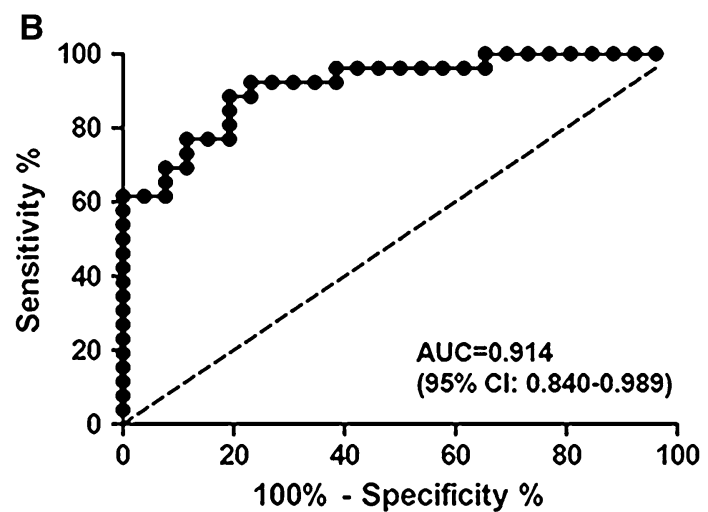

amplicon (a). The bars indicate the median with interquartile range. **indicates $p<0.0001$; ns indicates not significant. A ROC analysis was performed to discriminate RA patients from healthy controls (b)

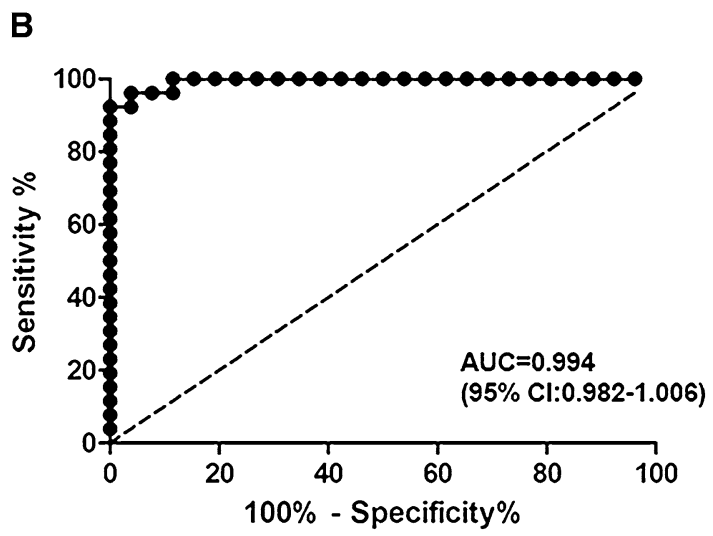

(a). The bars indicate the median with interquartile range. $* *$ indicates $p<0.0001$; ns indicates not significant. A ROC analysis was performed to discriminate RA patients from healthy controls (b)

samples (Fig. 5). Thus, the reduction of cfDNA levels appears to occur after disease onset.

\section{Serum DNase activity}

The reduced serum cfDNA levels in esRA might be explained by elevated levels and/or activity of nucleases present in the sera. Deoxyribonuclease I (DNase I) is one of the predominant DNases in human serum [24]. Deficiency in DNase I enzyme, and the resulting enhanced levels of cfDNA associated with nuclear antigens, promotes susceptibility to autoimmune disorders [25]. To investigate whether the reduced serum cfDNA levels were due to enhanced DNase activity in the sera, a radial enzyme diffusion method was carried out with sera from healthy controls $(n=50)$ and from esRA $(n=16)$ and RRMS $(n=44)$ patients. The sera were loaded on a gel containing 

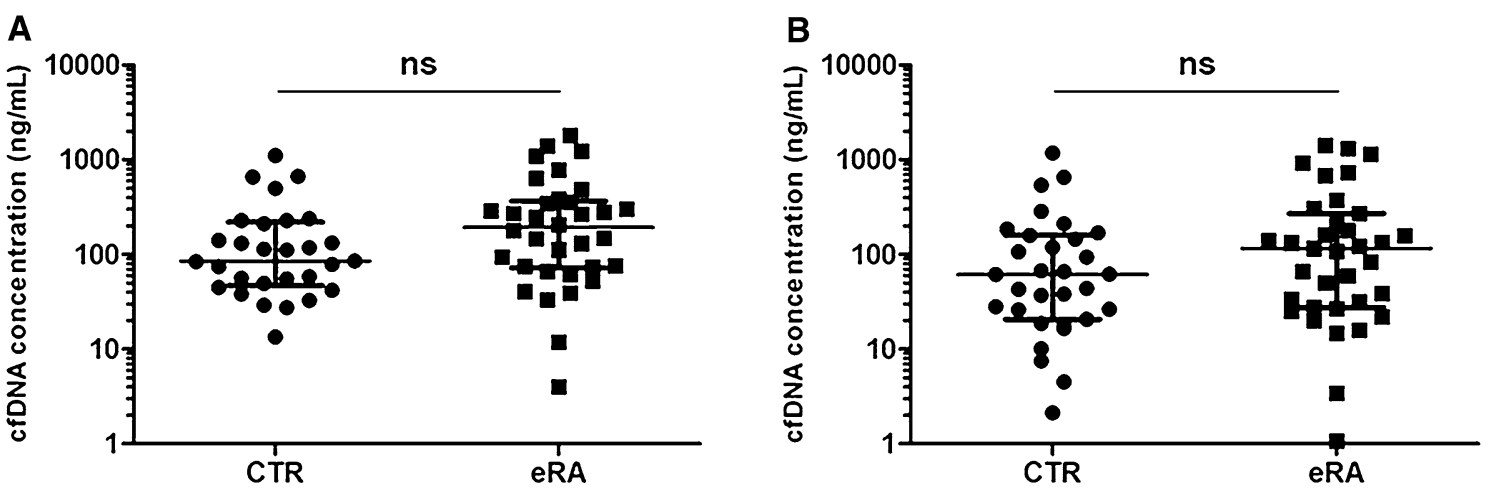

Fig. 4 cfDNA in early RA. DNA isolated from sera of healthy subjects (CTR) and eRA patients was analysed by qPCR using Alu115 primers (a) or $\beta$-actin205 primers (b). ns indicates not significant
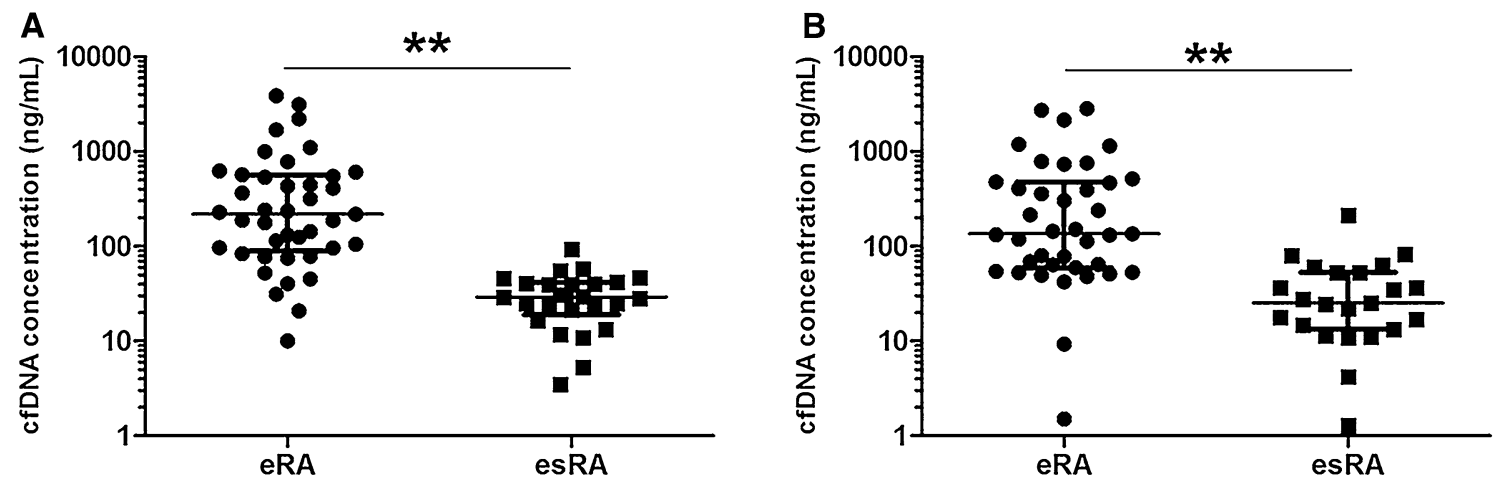

Fig. 5 Comparison of cfDNA concentration in sera of eRA and esRA patients. Serum cfDNA was purified and analysed by qPCR using Alu115 primers (a) or $\beta$-actin205 primers (b)

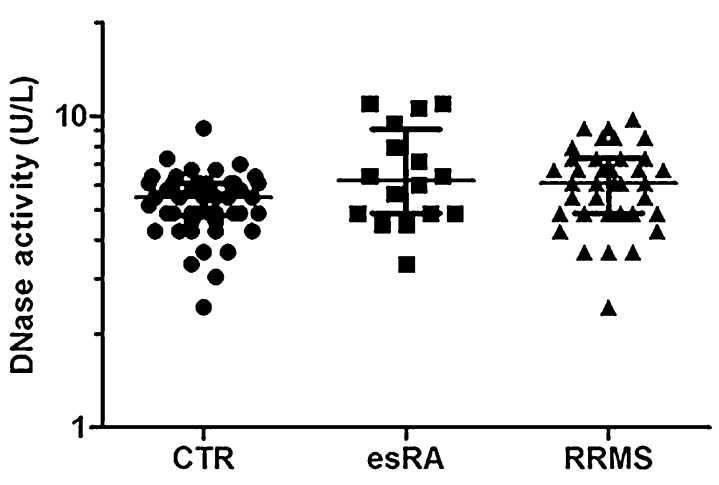

Fig. 6 DNase activity in sera from healthy individuals (CTR), RA and RRMS patients. The SRED method was used to determine the DNase activity in the sera. Sera were applied to wells in a $1.4 \%$ agarose gel containing DNA and a DNA-staining dye and incubated in a humidified chamber at $37{ }^{\circ} \mathrm{C}$ for $24 \mathrm{~h}$. Subsequently, the radius of the non-fluorescent area surrounding each well was measured

DNA, and, after incubation at $37^{\circ} \mathrm{C}$, DNA hydrolysis by nucleases present in the sera was visualized using a fluorescent dye. The results showed that no significant differences in total DNase activity between these groups were observed (Fig. 6). Thus, the reduced levels of cfDNA in
esRA sera do not seem to be due to elevated DNase activities.

\section{Discussion}

In the present study, we found reduced levels of serum cfDNA in established RA compared to healthy controls, early RA patients and RRMS patients. Lower levels of cfDNA have been reported before to occur in sera from SLE as well as in sera from RA patients in comparison with healthy subjects [26]. In addition, when nucleosome plasma levels were assessed by ELISA, nucleosomes appeared to be hardly detectable in the plasma of patients with RA in comparison with SLE patients [27]. Paradoxically, other studies showed a substantial increase in cfDNA concentration in plasma or serum of RA patients [16, 17, 28]. Leon et al. [28] reported that relatively high levels of cfDNA were found in patients with more severe symptoms who had active RA for less than 10 years, whereas patients with longer duration of disease showed a lower level of cfDNA. Elevated DNA levels were found more frequently in patients seronegative for rheumatoid factor (RF). Zhong 
et al. [17] found higher concentrations of cfDNA in serum and plasma of RA patients in comparison with healthy controls. These conflicting data on serum cfDNA levels in RA may at least in part be explained by the use of different techniques (for example, qPCR, ELISA or UV-spectrophotometry) and the different DNA targets chosen to perform the analyses. The PCR assay measures only amplifiable DNA, i.e., DNA fragments with a sufficient size and devoid of simple sequence repeats, whereas the assays based on spectrophotometry detect the total nucleic acid content such as dsDNA, ssDNA, and RNA. In addition, differences in the state of disease progression and/or activity at the time of sampling may have affected the results, as indicated by the differences observed for eRA and esRA. Moreover, differences in sample processing and storage might have introduced preanalytical biases. However, Sjoholm et al. [29] demonstrated that plasma and serum samples that were stored for 10-30 years produced reliable results when the cfDNA was extracted and amplified with PCR. In this respect we should also stress that samples collected in different centers were used in our study, which reduces the chance for such biases. Nevertheless, replication studies with distinct clinical cohorts are needed to support our findings. In addition, the analysis of larger groups may increase the statistical significance of our data.

We also observed slight overrepresentation of DNA quantified by the Alu repeats in sera in comparison with DNA quantified by the $\beta$-actin qPCR (see Fig. 3). However, the Alu115 and $\beta$-actin205 concentrations were highly correlated with each other for all groups tested (Supplementary Fig. S1). Our results are in agreement with previously reported observations. The proportion of Alu repeats relative to the $\beta$-globin gene was larger in serum DNA than in lymphocyte DNA, both in control subjects and in cancer patients [30]. In addition, overrepresentation of Alu repeats in serum cfDNA, in comparison with the complete human genome, was shown before for 50 healthy individuals [31]. The consistently higher levels of qPCRquantified DNA resulting from the Alu repeat analysis compared to the $\beta$-actin gene might be due to the fragmentation of DNA in serum. The chance that a complete Alu115 amplicon is present in a single DNA fragment is not only higher as a result of its size (115 basepairs compared to 205 basepairs for $\beta$-actin), but also due to the presence of multiple copies of the Alu115 amplicon in the human genome.

The exact mechanism by which DNA is released in blood is unknown. Previously, it has been demonstrated that the DNA in serum/plasma is predominantly hematopoietic in origin [32]. Four processes that may lead to the generation of circulating DNA have been suggested: cell death (necrosis or apoptosis) or tissue injury, active secretion of DNA by leukocytes, enucleation (a process in which the nuclei are expelled by erythroblasts during the course of maturation) and NETosis (a process in which neutrophils discharge extracellular traps).

Because we observed reduced levels of cfDNA in esRA patients, but not in eRA patients, it is tempting to speculate about a role for drug treatment. Methotrexate (MTX) is the most common disease-modifying anti-rheumatic drug (DMARD) used to treat RA. It reduces inflammation and inhibits enzymes involved in nucleoside/nucleotide metabolism. Previously, it has been shown that patients with RA, treated with MTX, display low concentrations of circulating purines and pyrimidines, with consequent reduced availability for DNA and RNA synthesis and cell proliferation [33]. Therefore, it will be very interesting to investigate whether the decrease of cfDNA in sera of esRA patients correlates with MTX treatment and dosage. In this respect, it is also important to note that the RRMS patients, which are only infrequently treated with MTX (only as a second line treatment), do not show altered cfDNA levels. None of the RRMS patients analyzed received MTX. The following processes might also cause the reduced levels of cfDNA in esRA patients: (1) increase of cfDNA degradation/elimination due to increased levels of circulating nuclease activity. However, we did not observe differences in the activity of serum nucleases between eRA and esRA patients and control individuals. (2) Increase of cfDNA degradation/elimination due to increased levels of serum anti-dsDNA antibodies. These antibodies can influence the level of circulating nucleosomes. An inverse correlation between anti-dsDNA antibody levels and concentrations of circulating DNA in SLE patients was observed [26]. RA patients can also develop anti-dsDNA antibodies induced by anti-TNF $\alpha$ biological therapies. They are usually of low avidity and are detectable transiently after treatment [34]. (3) Increase of cfDNA degradation/elimination due to enhanced metabolic activity and the generation of reactive oxygen species (ROS) by blood cells. It has been shown that DNA degradation products are elevated in leukocytes and sera of RA patients [35]. Moreover, increased oxidative enzyme activity along with decreased antioxidant levels in sera and synovial fluid of RA patients was observed. Higher levels of ROS in sera of esRA patients can change cfDNA degradation and lead to decreased cfDNA concentrations in sera. (4) Decreased apoptosis/ necrosis of blood cells. Altered apoptosis susceptibility of peripheral blood mononuclear cells for various systemic autoimmune diseases has been previously shown. The presence of activated peripheral blood $\mathrm{T}$ lymphocytes with impaired apoptosis, resulting in long-lived $\mathrm{T}$ cells peripherally in RA was reported [36]. Reduced apoptosis has been also detected in synovial tissues from patients with RA [37]. (5) Different susceptibility to degradation of 
various forms of circulating DNA such as shed cells, apoptotic bodies, nucleosomes, other nucleoproteins, and free DNA which exists in serum/plasma. The various forms of cfDNA might differ between healthy control and RA patients and the fates of different cfDNA forms are unknown.

In conclusion, the quantitative changes in serum cfDNA may be helpful as disease progression markers. Potential correlations between cfDNA levels and clinical features, such as disease activity, erosiveness and response to treatment, still need to be explored. It will be interesting to investigate whether the changes in cfDNA levels are affected by drugs used to treat RA patients. To further explore these possibilities, longitudinal studies will be necessary to get more insight in the time course of the reduction of cfDNA levels and the effects of drugs on these changes.

Acknowledgments We thank Dr. A. den Broeder (St. Maartenskliniek, Nijmegen, The Netherlands), Dr. L. Van de Putte (Radboud University Nijmegen Medical Center, The Netherlands) and Dr. T. Westgeest (Catharina Hospital, Eindhoven, The Netherlands) for the collection of sera from esRA patients and Dr. C. Zwanikken (Multiple Sclerosis Center Nijmegen, The Netherlands) for providing sera from RRMS patients. This work was supported in part by the Dutch Arthritis Association (Grant 12-2-203 to GJMP).

\section{Conflict of interest None.}

Informed consent Informed consent was obtained from all individual participants included in the study.

Ethical approval All procedures performed in studies involving human participants were in accordance with the ethical standards of the institutional and/or national research committee and with the 1964 Helsinki Declaration and its later amendments or comparable ethical standards.

\section{References}

1. van Venrooij WJ, van Beers J, Pruijn GJ (2011) Anti-CCP antibodies: the past, the present and the future. Nat Rev Rheumatol 7:391-398

2. Steiner G (2007) Auto-antibodies and autoreactive T-cells in rheumatoid arthritis: pathogenetic players and diagnostic tools. Clin Rev Allergy Immunol 32:23-36

3. Niienhuis S, Zendman A, Vossenaar E, Pruijn G, van Venrooij W (2004) Autoantibodies to citrullinated proteins in rheumatoid arthritis: clinical performance and biochemical aspects of an RAspecific marker. Clin Chim Acta 350:17-34

4. Landewe R (2003) The benefits of early treatment in rheumatoid arthritis: confounding by indication, and the issue of timing. Arthritis Rheum 48:1-5

5. Gauthier V, Tyler L, Mannik M (1996) Blood clearance kinetics and liver uptake of mononucleosomes in mice. J Immunol 156:1151-1156

6. Lo Y, Zhang J, Leung T, Lau T, Chang AM, Hjelm NM (1999) Rapid clearance of fetal DNA from maternal plasma. Am J Hum Genet 64:218-224
7. Rainer T, Wong L, Lam W, Yuen E, Lam NY, Metreweli C et al (2003) Prognostic use of circulating plasma nucleic acid concentrations in patients with acute stroke. Clin Chem 49:562-569

8. Geiger S, Holdenrieder S, Stieber P, Hamann GF, Bruening R, Ma J et al (2006) Nucleosomes in serum of patients with early cerebral stroke. Cerebrovasc Dis 21:32-37

9. Chang C, Chia R, Wu T, Tsao K, Sun C, Wu J (2003) Elevated cell-free serum DNA detected in patients with myocardial infarction. Clin Chim Acta 327:95-101

10. Rhodes A, Wort S, Thomas H, Collinson P, Bennett E (2006) Plasma DNA concentration as a predictor of mortality and sepsis in critically ill patients. Crit Care 10:R60

11. Gornik I, Wagner J, Gasparović V, Lauc G, Gornik O (2009) Free serum DNA is an early predictor of severity in acute pancreatitis. Clin Biochem 42:38-43

12. Schwarzenbach H, Hoon D, Pantel K (2011) Cell-free nucleic acids as biomarkers in cancer patients. Nat Rev Cancer 11:426-437

13. Dawson S, Tsui D, Murtaza M, Biggs H, Rueda OM, Chin SF et al (2013) Analysis of circulating tumor DNA to monitor metastatic breast cancer. N Engl J Med 368:1199-1209

14. Perkins G, Yap T, Pope L, Cassidy AM, Dukes JP, Riisnaes R et al (2012) Multi-purpose utility of circulating plasma DNA testing in patients with advanced cancers. PLoS One 7:e47020

15. Galeazzi M, Morozzi G, Piccini M, Chen J, Bellisai F, Fineschi S et al (2003) Dosage and characterization of circulating DNA: present usage and possible applications in systemic autoimmune disorders. Autoimmun Rev 2:50-55

16. Bartoloni E, Ludovini V, Alunno A, Pistola L, Bistoni O, Crino L et al (2011) Increased levels of circulating DNA in patients with systemic autoimmune diseases: a possible marker of disease activity in Sjögren's syndrome. Lupus 20:928-935

17. Zhong X, von Mühlenen I, Li Y, Kang A, Gupta AK, Tyndall A et al (2007) Increased concentrations of antibody-bound circulatory cell-free DNA in rheumatoid arthritis. Clin Chem 53:1609-1614

18. de Rooy D, van der Linden M, Knevel R, Huizinga T, van der Helm-van Mil A (2011) Predicting arthritis outcomes-what can be learned from the Leiden early arthritis clinic? Rheumatology 50:93-100

19. Umetani N, Kim J, Hiramatsu S, Reber H, Hines O, Bilchik A (2006) Increased integrity of free circulating DNA in sera of patients with colorectal or periampullary cancer: direct quantitative PCR for ALU repeats. Clin Chem 52:1062-1069

20. Huang Z, Hua D, Hu Y, Cheng Z, Zhou X, Xie Q et al (2012) Quantitation of plasma circulating DNA using quantitative PCR for the detection of hepatocellular carcinoma. Pathol Oncol Res $18: 271-276$

21. Chitrabamrung S, Bannett J, Rubin R, Tan E (1981) A radial diffusion assay for plasma and serum deoxyribonuclease I. Rheumatol Int 1:49-53

22. Ohmura K, Terao C, Maruya E, Katayama M, Matoba K, Shimada $\mathrm{K}$ et al (2010) Anti-citrullinated peptide antibody-negative RA is a genetically distinct subset: a definitive study using only bone-erosive ACPA-negative rheumatoid arthritis. Rheumatology 49:2298-2304

23. Seegobin S, Ma M, Dahanayake C, Cope AP, Scott DL, Lewis $\mathrm{CM}$ et al (2014) ACPA-positive and ACPA-negative rheumatoid arthritis differ in their requirements for combination DMARDs and corticosteroids: secondary analysis of a randomized controlled trial. Arthritis Res Ther 16:R13

24. Shakhmaeva I, Saifullina D, Sattarova L, Abdullin T (2011) Electrochemical sensor for blood deoxyribonucleases: design and application to the diagnosis of autoimmune thyroiditis. Anal Bioanal Chem 401:2591-2597 
25. Napirei M, Ricken A, Eulitz D, Knoop H, Mannherz H (2004) Expression pattern of the deoxyribonuclease 1 gene: lessons from the Dnase1 knockout mouse. Biochem J 380:929-937

26. Jørgensen M, Rekvig O, Jacobsen R, Jacobsen S, Fenton K (2011) Circulating levels of chromatin fragments are inversely correlated with anti-dsDNA antibody levels in human and murine systemic lupus erythematosus. Immunol Lett 138:179-186

27. D'Auria F, Rovere-Querini P, Giazzon M, Ajello P, Baldissera E, Manfredi AA et al (2004) Accumulation of plasma nucleosomes upon treatment with anti-tumour necrosis factor-alpha antibodies. J Intern Med 255:409-418

28. Leon S, Ehrlich G, Shapiro B, Labbate V (1977) Free DNA in the serum of rheumatoid arthritis patients. J Rheumatol 4:139-143

29. Sjoholm M, Hoffmann G, Lindgren S, Dillner J, Carlson J (2005) Comparison of archival plasma and formalin-fixed paraffin-embedded tissue for genotyping in hepatocellular carcinoma. Cancer Epidemiol Biomark Prev 14:251-255

30. Beck J, Urnovitz HB, Riggert J, Clerici M, Schütz E (2009) Profile of the circulating DNA in apparently healthy individuals. Clin Chem 55:730-738

31. Stroun M, Lyautey J, Lederrey C, Mulcahy HE, Anker P (2001) Alu repeat sequences are present in increased proportions compared to a unique gene in plasma/serum DNA: evidence for a preferential release from viable cells? Ann N Y Acad Sci 945:258-264

32. Lui Y, Chik K, Chiu R, Ho C, Lam C, Lo Y (2002) Predominant hematopoietic origin of cell-free DNA in plasma and serum after sex-mismatched bone marrow transplantation. Clin Chem 48:421-427

33. Smoleńska Z, Kaznowska Z, Zarówny D, Simmonds HA, Smoleński R (1999) Effect of methotrexate on blood purine and pyrimidine levels in patients with rheumatoid arthritis. Rheumatology 38:997-1002

34. Hyrich K, Silman A, Watson K, Symmons D (2004) Anti-tumour necrosis factor alpha therapy in rheumatoid arthritis: an update on safety. Ann Rheum Dis 63:1538-1543

35. Hitchon C, El-Gabalawy H (2004) Oxidation in rheumatoid arthritis. Arthritis Res Ther 6:265-728

36. Szodoray P, Jellestad S, Nakken B, Jg Brun, Jonsson R (2003) Programmed cell death in rheumatoid arthritis peripheral blood T-cell subpopulations determined by laser scanning cytometry. Lab Invest 83:1839-1848

37. Smith M, Weedon H, Papangelis V, Walker J, Roberts-Thomson P, Ahern M (2010) Apoptosis in the rheumatoid arthritis synovial membrane: modulation by disease-modifying anti-rheumatic drug treatment. Rheumatology 49:862-875 